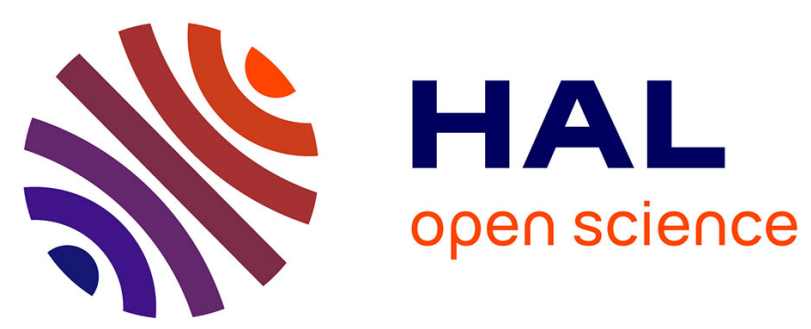

\title{
Quantum size effects in the inner crust of neutron stars
}

F Barranco, R A Broglia, E Vigezzi

\section{To cite this version:}

F Barranco, R A Broglia, E Vigezzi. Quantum size effects in the inner crust of neutron stars. Journal of Physics G: Nuclear and Particle Physics, 2010, 37 (6), pp.64023. 10.1088/0954-3899/37/6/064023 . hal-00600807

\section{HAL Id: hal-00600807 https://hal.science/hal-00600807}

Submitted on 16 Jun 2011

HAL is a multi-disciplinary open access archive for the deposit and dissemination of scientific research documents, whether they are published or not. The documents may come from teaching and research institutions in France or abroad, or from public or private research centers.
L'archive ouverte pluridisciplinaire $\mathbf{H A L}$, est destinée au dépôt et à la diffusion de documents scientifiques de niveau recherche, publiés ou non, émanant des établissements d'enseignement et de recherche français ou étrangers, des laboratoires publics ou privés. 


\title{
Quantum size effects in the inner crust of neutron stars
}

\author{
F. Barranco \\ Departamento de Fisica Aplicada III, Universidad de Sevilla, \\ Escuela Superior de Ingenieros, 41092 Sevilla, Spain \\ E-mail: barranco@us.es
}

\author{
R.A. Broglia \\ INFN, Sezione di Milano, 20133 Milano, Italy \\ The Niels Bohr Institute, 2100 Copenhagen Ø, Denmark \\ E-mail: broglia@mi.infn.it \\ E. Vigezzi \\ INFN, Sezione di Milano, 20133 Milano, Italy \\ E-mail: vigezzi@mi.infn.it
}

Dipartimento di Fisica, Università di Milano, 20133 Milano, Italy

\begin{abstract}
It is generally accepted that the inner crust of neutron stars is formed by a Coulomb lattice of nuclei immersed in a gas of quasi-free neutrons. We discuss the implications of the inhomogeneity of the crust and of nuclear shell effects on the linear response and on the superfluid properties of the system, as well as on the structure of vortices.
\end{abstract}

\section{Introduction}

The first study of the structure of the inner crust of neutron stars based on detailed quantum calculations was the paper published by Negele and Vautherin in 1973 [1]. Their work was truly ahead of their times, as is demonstrated by the fact that it has received about two thirds of its about 400 citations after the year 1995, and only about $10 \%$ in the decade following its publication [2]. This reflects the theoretical effort deployed in recent times towards a detailed understanding of the complex physics of the crust. It also indicates that the basic structure of the crust as described by Negele and Vautherin - a Coulomb lattice formed by spherical nuclei immersed in a sea of quasi-free neutrons, with a lattice step that decreases going towards the core of the star - is still valid today, at least for nucleon densities up to about $0.03 \mathrm{fm}^{-3}$. The precise determination of the density range in which spherical nuclei are energetically favoured, as compared to other phases with different spatial structures, is an open and important 
issue. Also the isotopic composition of the crust depends on the details of the calculation and in particular on pairing correlations [3]. For a recent, general review of the physics of the inner crust, we refer to [4].

In the conclusions of their study, Negele and Vautherin remarked that 'the degree to which the nuclei in the free neutron regime resemble ordinary nuclei' is striking, and that 'this similarity is also manifested in the behaviour of the single-particle energies'. In the present contribution, various issues associated with the persistence of the shell structure in the low-density part of the inner crust will be discussed. Of notice that shell effects have mostly been considered in connection with the calculation of ground state energies. Here, we shall focus on the role of inhomogeneities and quantal finite-size effects concerning the linear response and the superfluidity of the crust. These effects turn out to be particularly important in the microscopic description of vortices, which, arguably, are the most direct manifestation of superfluidity of the crust in a rotating pulsar, as in atomic Fermi gases [5].

\section{Shell effects and linear response}

Going from the surface towards the center of a neutron star, the separation energy of neutrons from nuclei diminishes, until at densities larger than about $4 \times 10^{11} \mathrm{~g} \mathrm{~cm}^{-3}$, neutrons start to drip out of nuclei and to occupy states in the continuum part of the spectrum. This defines the beginning of the inner crust, which extends over a density range of about three orders of magnitude, until the core of the star is reached for densities of the order of $1.4 \times 10^{14} \mathrm{~g} \mathrm{~cm}^{-3}\left(\right.$ or $0.08 \mathrm{fm}^{-3}$ ), and neutron star matter becomes homogeneous.

Many studies have addressed the structure of the inner crust, using the liquid drop approximation, semiclassical methods, Hartree-Fock quantum calculations or molecular dynamics simulations. A Coulomb lattice of spherical nuclei appears generally to be the favoured phase up to about $0.03 \mathrm{fm}^{-3}$. Most calculations in this phase have been based on the Wigner-Seitz (WS) approximation, in which one deals with a single elementary cell of the Coulomb lattice, approximating it with a sphere. One then minimizes the energy of neutron, proton and electron matter in $\beta$-equilibrium within the WS cell, whose radius is considered as a variational parameter, determining the isotopic composition of the cell. According to the few studies which have taken the band structure of the Coulomb lattice into account $[6,7]$, the WS approximation turns out to be rather reliable, but this is a topic that should be further investigated, in particular when the radius of the WS cell becomes smaller than about $20 \mathrm{fm}$ and the results of calculations show a marked dependence on the specific boundary conditions [8].

In the following we shall present results obtained in a WS cell of radius $R_{W S}=33.1$ fm, containing 1314 neutrons and with $Z=50$ protons at the center. This cell is representative of nucleon density $n \approx 1.5 \times 10^{13} \mathrm{~g} / \mathrm{cm}^{3}\left(n \approx 0.01 \mathrm{fm}^{-3}\right)$.

In Fig. 1 we show the neutron and proton mean fields, as well as the neutron and 

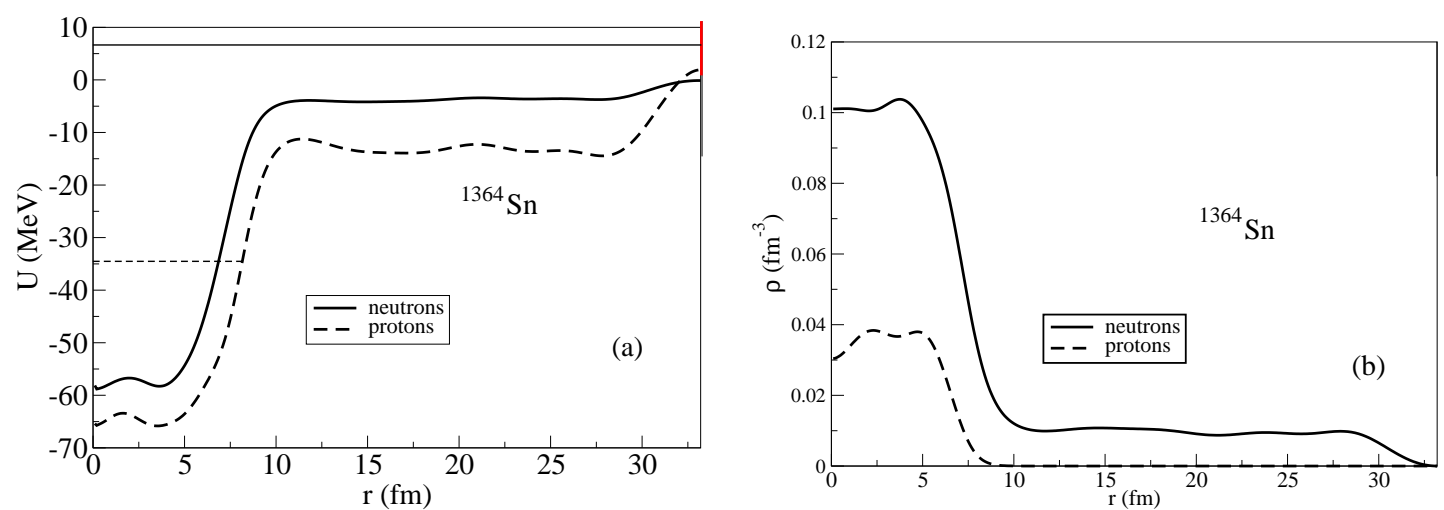

Figure 1. Neutron and proton mean field potentials (a) and densities (b) in the ${ }^{1364} \mathrm{Sn}$ Wigner-Seitz cell containing 50 protons and 1314 neutrons. The thin solid and dashed lines in (a) indicate the neutron and proton Fermi energies.

proton densities resulting from a self-consistent, spherical HF calculation performed with the effective interaction SLy4, imposing that the wavefunctions vanish at the edge of the cell . The neutron and proton Fermi energies lie at $E_{F}=5.5 \mathrm{MeV}$ and $-35.1 \mathrm{MeV}$ respectively. Notice that the neutron continuum spectrum starts at $E \approx-3.5 \mathrm{MeV}$, and that the bound neutron single-particle levels are occupied by about 130 neutrons.

In the HF calculation, we deal with discrete states in the spherical WS cell, and the potential goes to zero at the edge of the cell. It proves of interest to extrapolate the HF potential of the WS cell from the wide central region where it is about constant up to infinity. The energy dependence of the phase shifts calculated in the extrapolated neutron potential is shown in Fig. 2. One finds several resonant single-particle states in the continuum, corresponding to values of the orbital angular momentum $l$ up to 10. The centroid and the width of the resonances are given in Table 1 . The centroid is taken to be the equal to the energy at which the phase shift $\delta_{l j}(E)$ reaches the value $\pi / 2$ or $3 \pi / 2$ with positive slope, while the width is obtained from the full width at half maximum of the function $d \delta_{l j}(E) / d E$.

As displayed in Table 1, some of the states have a rather narrow width, of the order of a few $\mathrm{MeV}$. The spacing between resonances in ${ }^{1364} \mathrm{Sn}$ corresponds to a typical oscillator frequency $\hbar \omega \approx 8 \mathrm{MeV}$, a value which is not very different from that typical of a heavy nucleus of mass number $A \approx 200$ (that is, with a similar number of bound nucleons), according to the standard estimate $\hbar \omega \approx 41 A^{-1 / 3} \mathrm{MeV}$. These resonances can play an important role in different contexts. In particular, they determine the linear response of the nucleus immersed in the neutron sea.

In Fig. 3 the particle-hole transitions which are associated with the largest values of the strength of the quadrupole and octupole operators $d U / d r Y_{20}$ and $d U / d r Y_{30}$ are displayed; $U$ denotes the local neutron HF field associated with the zero-range SLy4 force (similar results would be obtained using the neutron density instead of $U$ ). In all cases, they involve unbound states whose quantum numbers $\{l, j\}$ correspond to those reported in Table 1, and whose energies lie close to those of the corresponding centroids. Easy 

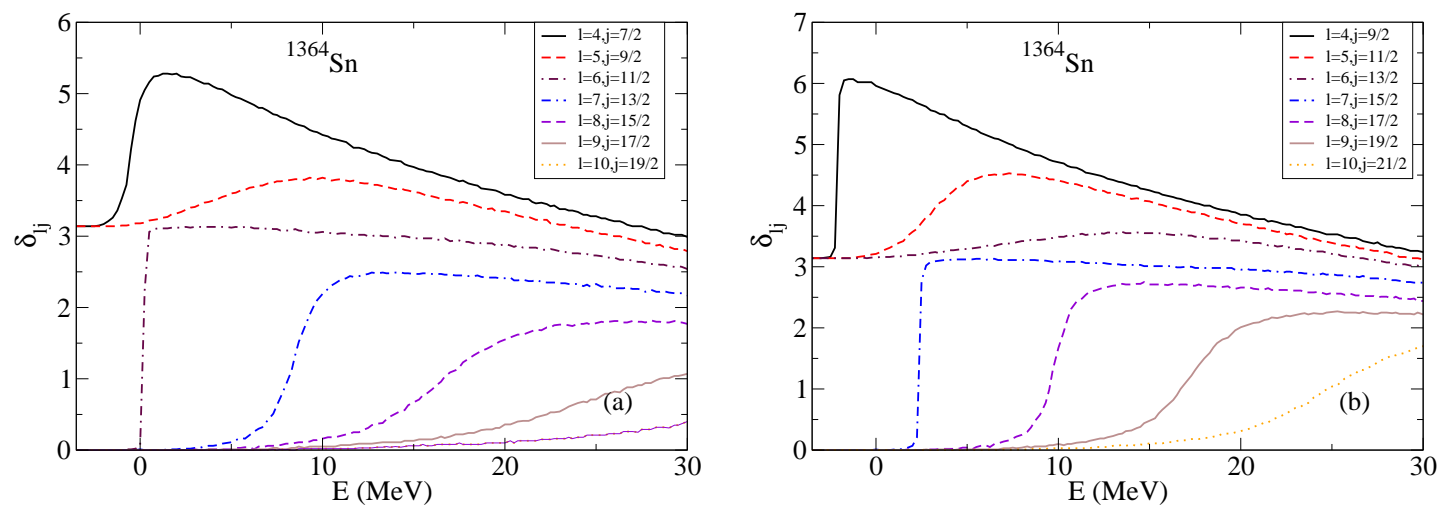

Figure 2. Phase shifts calculated as a function of energy for different values of the total and orbital angular momentum of the neutron single-particle states in the exrtapolated HF potential of the ${ }^{1364} \mathrm{Sn}$ WS cell. The phase shifts associated with spin down $(j=l-1 / 2)$ and spin up $(j=l+1 / 2)$ neutron single-particle orbitals are reported in (a) and (b) respectively. The continuum spectrum starts at $E \approx-3.5 \mathrm{MeV}$ (cf. Fig. $1)$.

\begin{tabular}{c|c|c|c}
\hline$l$ & $j$ & $E_{l j}^{r e s}[\mathrm{MeV}]$ & $\Gamma_{l j}^{r e s}[\mathrm{MeV}]$ \\
\hline 4 & $9 / 2$ & -2.1 & 0.08 \\
4 & $7 / 2$ & -0.2 & 1.0 \\
6 & $11 / 2$ & 0.2 & 0.04 \\
7 & $15 / 2$ & 2.4 & 0.08 \\
7 & $13 / 2$ & 8.7 & 2.5 \\
8 & $17 / 2$ & 9.9 & 1.7 \\
8 & $15 / 2$ & 20.3 & 8.0 \\
9 & $19 / 2$ & 18.3 & 4.5 \\
10 & $21 / 2$ & 28.7 & 9.0 \\
\hline
\end{tabular}

Table 1. Total and orbital angular momentum $l j$, energies $E_{\text {res }}$ and widths $\Gamma_{\text {res }}$ of the resonant neutron single particle states calculated extrapolating the HF potential of the ${ }^{1364}$ Sn WS cell up to infinity (cf. Fig. 1 and 2).

to recognize are low-lying octupole transitions, having an energy approximately given by $\Delta E \approx \hbar \omega$, and high-lying quadrupole and octupole transitions having $\Delta E \approx 2 \hbar \omega$ and $\Delta E \approx 3 \hbar \omega$ respectively. In other words, the typical shell structure of the response observed in atomic nuclei is preserved in the inner crust, as can be seen in Fig. 4, where the octupole strength calculated in the Random Phase Approximation (RPA) is displayed. It is found (see ref. [9]) that the strength evolves in a rather continuous fashion going from atomic nuclei into the inner crust. These results should be contrasted with those obtained making use of the operator $r^{\lambda}$, which heavily weight the free neutron part of the cell. In that case, the strength associated with the response of bound or resonant states is overwhelmed by the response of the free neutrons $[10,11]$. 


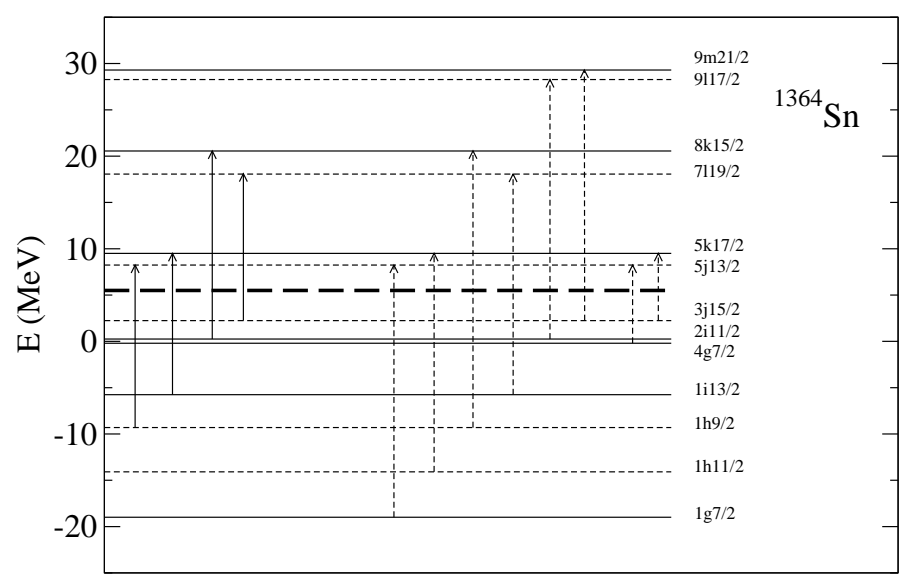

Figure 3. Neutron particle-hole transitions associated with the largest values of the strength of the operator $d U / d r Y_{20}$ and $d U / d r Y_{30}$ in the WS cell ${ }^{1364} \mathrm{Sn}$. Neutron single-particle levels of even and odd parity are drawn by solid and dashed lines, and are labeled by the usual spherical quantum numbers $\{n l j\}$. Solid and dashed arrows refer to $2^{+}$and to $3^{-}$transitions. The neutron Fermi energy is represented by the thick dashed line.

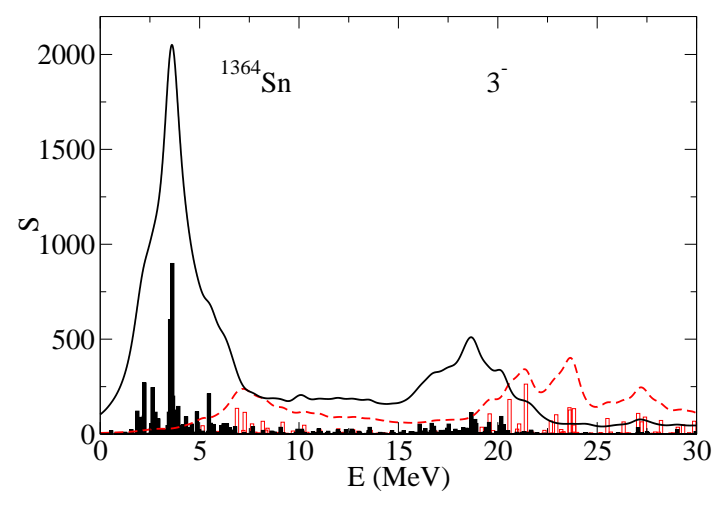

Figure 4. Strength functions associated with the octupole operator $d U / d r Y_{30}$ in the ${ }^{1364} \mathrm{Sn}$ WS cell. The discrete unperturbed and RPA responses are shown by the open and filled histograms (in units of $\mathrm{MeV}^{2} \mathrm{fm}^{-2}$ ). The dashed and solid curves (in units of $\mathrm{MeV} \mathrm{fm}^{-2}$ ) are obtained folding the unperturbed and the RPA discrete response with a Lorentzian curve having a width of $1 \mathrm{MeV}$.

\section{Pairing correlations in the Wigner-Seitz cell}

On the basis of BCS theory and using standard nucleon-nucleon potentials, one finds that neutron matter in the ${ }^{1} S_{0}$ channel is superfluid at the densities and at the temperatures $(T \approx 0.01 \mathrm{MeV})$ typical of the inner crust. If one ignores its inhomogeneities, the inner crust can be considered as a macroscopic piece of neutron matter, where superfluid flow can fully develop. At a density $n \approx 0.01 \mathrm{fm}^{-3}$ (corresponding to a Fermi momentum $k_{F} \approx 0.65 \mathrm{fm}^{-1}$ ) one would estimate a value $\xi \approx \hbar^{2} k_{F} / m \pi \Delta \approx 6.5 \mathrm{fm}$ for the coherence length, using the value $\Delta \approx 2 \mathrm{MeV}$ calculated in uniform matter at this density with the bare nucleon-nucleon force. This is smaller than the coherence length in the atomic nucleus ( $\xi \approx 20 \mathrm{fm}$, using $\Delta \approx 1 \mathrm{MeV}$ and $k_{F} \approx 1.5 \mathrm{fm}^{-1}$ ), where however superflow is 
limited by the small value of the nuclear radius.

The typical values of the coherence length in the inner crust are several times larger than the diffusivity of the nuclear potential, and the application of the results obtained in neutron matter to the actual case of the inner crust requires a careful consideration of its inhomogeneous character. Pethick and Ravenhall wrote in 1995 that since 'the spatial variation of superfluid gaps is not well understood theoretically, even in the absence of superfluid flow, the properties of vortices in such an inhomogeneous medium are even less well understood from basic theory' [12]. Much progress has been made since then concerning pairing correlations. The spatial dependence of the pairing gap has been studied by several groups at the mean field level, using parametrized potentials or selfconsistent Hartree-Fock-Bogoliubov (HFB) theory [13, 14, 15, 16, 17, 18]. According to the Local Density Approximation (LDA), one expects that the gap should be suppressed in the interior of the nucleus, because there the local Fermi momentum is usually larger than in the external neutron gas. The effect is reduced in actual HFB calculations, because LDA does not take into account proximity effects: close to the nuclear surface, a Cooper pair feels both the interior of the nuclear impurity and the external free neutron superfluid. As a result, the value of the gap close to the Fermi energy is decreased by a few hundred $\mathrm{keV}$ compared to the value in neutron matter, leading to an increase of the specific heat of the crust which can affect the earliest phases of neutron star cooling, at least in some astrophysical scenarios $[15,17,19]$. Nonetheless, the influence of the inhomogeneities on the pairing gap at the mean field level is considerably smaller than typical uncertainties adscribed to the existing calculations of medium polarization effects.

Within this context one can mention the fact that two 'ab initio' calculations of the gaps in uniform neutron matter carried out recently with the help of Montecarlo techniques $[20,21,22]$ are found to be in rather poor agreement with each other. Furthermore, one is still lacking a critical comparison and assessment of the differences existing between these calculations and studies based on the renormalization group [23] or on Brueckner and Nambu-Gorkov theory [24, 25], in particular concerning the role played by collective modes.

In spite of quantitative differences, all these calculations predict a reduction of the pairing gap as compared to the BCS results. This is because polarization effects in neutron matter are dominated by the repulsion induced by spin fluctuations. This is different from what is found in atomic nuclei, in which the exchange of collective, low-lying surface modes between Cooper pair partners induces an attractive pairing interaction which enhances the gap. Also in this case, the quantitative aspects of such results are lively debated $[26,27,28,29]$.

The inhomogeneous WS cell poses a particularly interesting challenge, due to the interplay of the free neutron and of the nuclear phase. Some calculations have used a density functional obtained interpolating between a functional adopted in neutron matter and a functional used in atomic nuclei [30]. Only exploratory calculations have directly addressed medium renormalization effects in the inner crust $[31,32]$. They 
have explicitly included the coupling with the vibrations of the systems in the WS cell (cf. Section 2), and indicate that the screening of the gap due to spin fluctuations is counterbalanced to some extent by density fluctuations associated with the dynamics of the surface of the nucleus. This leads to characteristic changes in the spatial dependence of the pairing gap close to the nuclear surface. Of notice that the effect of the vibrations of the Coulomb lattice on the pairing induced interaction has never been considered [33].

It would be important to have evidence of superfluidity in the crust directly associated with astronomical observations. As we have already mentioned, superfluidity affects the thermal properties of the crust. This can influence neutron star cooling, but simulations generally show that cooling is much more sensitive to the properties of the core [34]. However, recent observations of rapid cooling of the crust after accretion have open exciting perspectives, because this phenomenon directly depends on the properties of the crust. Simulations indicate that neutron superfluidity in the crust is indeed needed to obtain agreement with the data $[35,36]$.

\section{Vortex structure and pinning to nuclei}

In keeping with the ansatz that neutron star matter is superfluid, the rotation of the star should induce the formation of vortices in the inner crust.

While there is a rich literature concerning vortices in condensed matter, only few studies have been devoted to the quantum structure of vortices in neutron matter in general [37] and in neutron stars in particular [38, 39, 40, 41]. It turns out that shell structure can play an important role in a microscopic study of a vortex in the inner crust. This is because vortices are excitations based on Cooper pairs made out of single-particle states having different parity.

In what follows we shall only consider the axially symmetric case, in which the vortex axis coincides with the $z$-axis. The vortex can be characterized by a pairing field

$$
\Delta(\rho, z, \phi)=\Delta(\rho, z) e^{i \nu \phi}
$$

where the second factor depends only on the azimuthal angle $\phi$ about the $z$-axis. The index $\nu$ is the vortex number. It indicates the number of quanta of angular momentum carried by each Cooper pair. In particular, if we set $\nu=0$ we obtain the usual HFB equations (no vortex). Setting instead $\nu=1$, we can describe a vortex excitation, in which each Cooper pair carries one unit of angular momentum along the $z$-axis. Experiments on superfluids indicate that it is energetically more favorable to develop an array of $\nu=1$ vortices rather than a few vortices carrying many quanta of angular momentum. In the following we shall assume that this is also the case in neutron stars.

When the vortex axis goes through the center of one of the nuclei building the Coulomb lattice, it is said that the vortex is pinned. The associated pairing field is axially symmetric (cf. Fig. 5), in keeping with the spherical symmetry of the nuclear mean field. 
For a given value of $m$, the projection of the orbital angular momentum on the $z$-axis, and indicating the other quantum numbers by the index $q$, the quasiparticle amplitudes have the form

$$
\begin{aligned}
& v_{q m}(\rho, z, \phi)=V_{q m}(\rho, z) e^{i m \phi} \\
& u_{q m}(\rho, z, \phi)=U_{q m}(\rho, z) e^{i(m-\nu) \phi}
\end{aligned}
$$

with the property $V_{q, m}(\rho, z)=V_{q,-m+\nu}(\rho, z)$, which for $\nu=1$ leads to an azimuthal current given by

$$
J(\rho, z, \phi)=-\frac{i \hbar}{m \rho} \sum_{q m} v_{q m}^{*}(\rho, z, \phi) \frac{\partial v_{q m}(\rho, z, \phi)}{\partial \phi},
$$

with the associated velocity field $J / n$. For large values of $\rho$ Eq. (3) leads to the well known Onsager profile, $v=J / n=\hbar / 2 m \rho$.
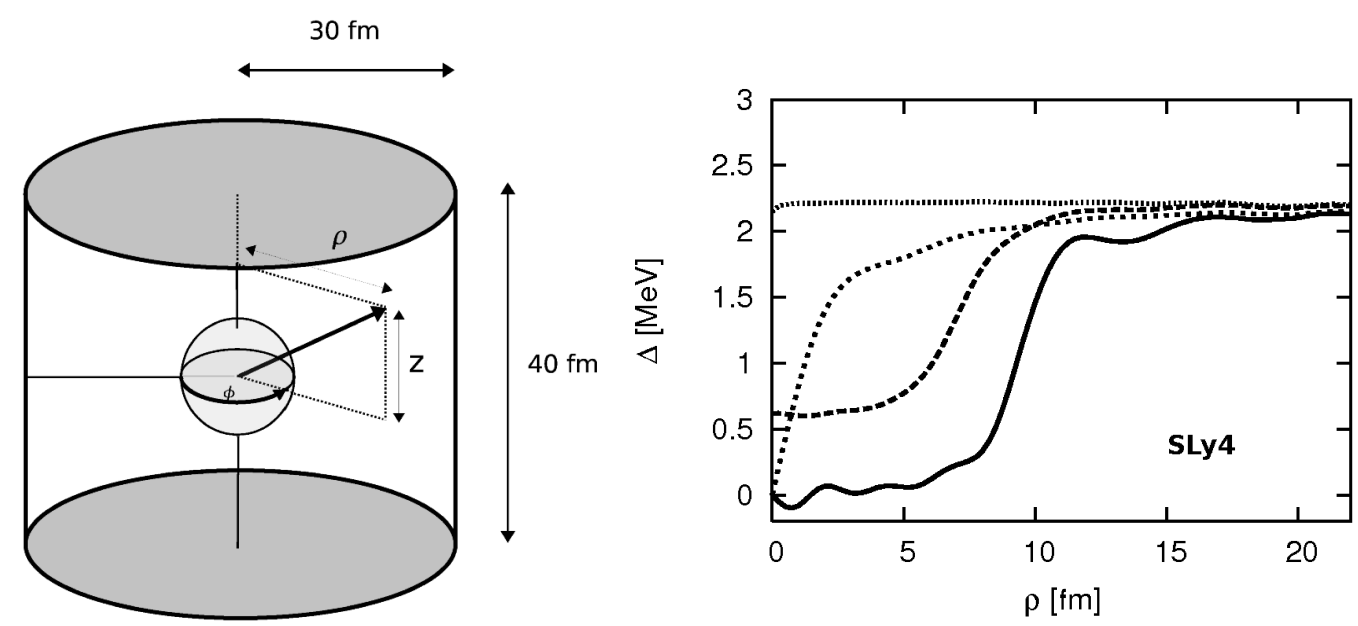

Figure 5. (left) Geometry used to calculate the pairing gaps shown in the right panel. (Right) Pairing gaps as a function of $\rho$ in the equatorial plane $(z=0)$ associated with four different configurations (vortex pinned on a nucleus in the WS cell : solid curve; vortex in uniform neutron matter: short dashed curve; nucleus in the WS cell: dashed curve; uniform neutron matter: dotted curve) calculated for an asymptotic neutron density $0.01 \mathrm{fm}^{-3}$ at large values of $\rho$.

Aside from axial symmetry, the system has mirror symmetry with respect to the $x-y$ plane (see Fig. 5 ), so that $\Delta(\rho, z)=\Delta(\rho,-z)$. Applying the parity operator $\hat{\Pi}$ $(\phi \rightarrow \phi+\pi$ and $z \rightarrow-z)$ to the pairing field one obtains

$$
\hat{\Pi} \Delta(\rho, z) e^{i \nu \phi}=e^{i \nu \pi} \Delta(\rho, z) e^{i \nu \phi},
$$

implying that for $\nu=1$ the Cooper pairs are constructed out of single-particle levels of opposite parity. As discussed in Section 2, the persistence of the shell structure in the WS cell implies the existence of resonant states of different parity, which are separated by an energy $\hbar \omega \approx 8 \mathrm{MeV}$. This hinders the formation of a vortex within the nuclear volume as compared to the uniform situation. The pairing gap associated with a vortex pinned on a spherical nucleus, obtained solving the HFB equations in the WS cell [41], 
is shown by the solid line in the right panel of Fig. 6, as a function of the distance from the vortex axis in the equatorial plane of the nucleus. Also displayed are the gaps calculated: (a) in the WS cell without a vortex $(\nu=0),(\mathrm{b})$ in the ground state of uniform neutron matter $(\nu=0)$, (c) for a vortex in uniform matter $(\nu=1)$. In all cases the same pairing force was used, namely, a density-dependent, zero-range interaction whose parameters are tuned to reproduce the gap obtained with the bare force.

It is seen that the gap of the pinned vortex is strongly suppressed not only in the interior of the nucleus, but also in a rather thick layer at the interface between the nucleus and the neutron gas. The vortex radius can be estimated as the distance from the $z$-axis at which $\Delta$ reaches a value equal to $90 \%$ of its asymptotic value. In the case displayed in Fig. 5, it has a value of about $12 \mathrm{fm}$, that is, about $6 \mathrm{fm}$ larger than in uniform matter (cf. the solid and the short-dashed curve).

An important issue is whether the vortex lines tend to be pinned to the nuclei forming the Coulomb lattice. If for some reason vortices were catastrophically unpinned from the nuclei, they could release angular momentum to the neutron star surface and the angular velocity of the star would show a sudden increase [42]. This might explain the irregularities (glitches) observed in the period of the radiation emitted by a large number of pulsars [43]. Consequently an important quantity which can be obtained from a microscopic study of the vortex structure is the pinning energy, namely, the difference between the energy of the system when the axis of the vortex goes through the center of the nucleus and when the axis of a vortex line is placed far from the nuclei.

According to semiclassical models $[44,45,46]$, the pinning energy reflects a competition between the kinetic energy flow and the condensation energy. In such a competition, the size of the vortex core, defining the region in which the gap vanishes, is generally smaller than the nuclear radius; then the pinning energy may be negative, vortices being attracted by nuclei. This is in keeping with the fact that the loss of condensation energy associated with the vortex core can be less severe if the vortex goes through the nucleus, where the gap was already small in the absence of the vortex, as compared to the outer neutron gas.

The large vortex radii found in the quantum calculations strongly influence the contribution of the condensation energy and of the flow energy to the pinning energy, invalidating the semiclassical approximation, and changing the dependence of the pinning energy on density in a qualitative way. In spite of the progress accomplished in the understanding of the basic vortex structure, at present it is still not possible to calculate a reliable value of the pinning energy. This is because of the uncertainties adscribed to the effective interactions, concerning not only the influence of medium polarization effects on the pairing interaction, but also the effective mass associated with the interaction used to calculate the mean field, a quantity which affects, among other properties, the spacing between resonances.

The strength of the interaction between a segment of a vortex line and a nucleus is one of the basic elements determining the vortex dynamics in the crust. The latter constitutes a complex problem (cf. Sect. 8.3 in [4] for a review), although it is simplified 
to some extent because vortices can safely assumed to be isolated, their radius (of the order of the coherence length) being much smaller than the intervortex distance (of the order of $10^{-4} \mathrm{~cm}$ for a millisecond pulsar). An attempt towards a detailed description of the interaction of a vortex line with the Coulomb lattice has been made recently [47]. The motion of the line is determined by the equations of classical mechanics including the force exerted by the nuclei of the lattice, the vortex tension, the hydrodynamical Magnus force which tends to push the vortex towards the surface of the star as well as a dissipative force that accounts for electron scattering and for the generation of vortex waves (Kelvin modes). In particular, the Magnus force depends on the relative velocity $v_{r e l}$ between the vortex line and the macroscopic superfluid flow produced by the ensemble of the vortices. For values of $v_{r e l}$ below a critical value, which is determined by the absolute value of the pinning energy, one finds that the vortex line is immobilized within the lattice. Above this velocity the vortex can move through the lattice towards the surface of the star where it disappears transferring its angular momentum to the non superfluid, rigid components of the star. Pinning energies of the order of $1 \mathrm{MeV}$ correspond to critical velocities of the order of $10^{6}-10^{7} \mathrm{~cm} / \mathrm{s}$, which allow the system to store enough angular momentum to drive a giant glitch.

The axial symmetry adopted in the previous discussion is appropriate for the interaction of a vortex with isolated, spherical nuclei. However, according to several calculations based on the semiclassical Thomas Fermi approximation, on quantum HF theory, or on molecular dynamics, in the deeper layers of the crust the stable nuclear configuration changes with increasing volume fraction of the nuclei, going through a sequence of shapes, from spheres to cylinders (spaghetti), slabs (lasagna), tubes and bubbles, until the homogeneous phase becomes favoured and the core of the star is reached (cf. Section 3.3 in [4]). The interaction between a vortex and one of these structures has never been computed microscopically (with the partial exception of the cylinder, cf. [39]). A systematic analysis would require full threedimensional calculations, which should explore different configurations, considering different alignments of the vortex lines with respect to the various shapes.

\section{Conclusions}

We have presented an overview of recent progress made in the study of the quantal structure of the inner crust of neutron stars. We have shown that quantum microscopic calculations are needed, to reveal qualitative effects associated with the inhomogeneous nature of the inner crust and neutron shell effects in the continuum. We have also indicated some of the areas where much work remains to be done, in particular concerning a quantitative treatment to deal with the effects associated with band structure, with the influence of medium polarization on superfluidity and with the connection between the microscopic structure of vortices and their dynamics in the star. 


\section{Acknowledgment}

This paper is based on work carried out in collaboration with P. Avogadro, S. Baroni, A. Pastore and F. Raimondi. F.B. acknowledges partial support from the Spanish Education and Science Ministry Project Nos. FPA2006-13807-c02-01, FIS2005-01105, and INFN08-33.

\section{References}

[1] Negele J and Vautherin D 1973 Nucl. Phys. A 207298

[2] Citation data are taken from ISI Web of Knowledge.

[3] Baldo M, Lombardo U, Saperstein EE and Tolokonnikov SV 2005 Nucl. Phys. A 750409

[4] Chamel N and Haensel P 2008 Living Rev. Relativity 1110

[5] Zwierlein MW, Abo-Schaerr JR, Schirotzek A, Schunck CH and Ketterle W 2005 Nature 4351047

[6] Chamel N 2005 Nucl. Phys. A 747109

[7] Chamel N, Naimi S, Khan E and Margueron J 2007 Phys. Rev. C 75055806

[8] Baldo M, Saperstein EE and Tolokonnikov SV 2006 Nucl. Phys. A 775235

[9] Baroni S, Pastore A, Raimondi F, Barranco F, Broglia RA and Vigezzi E, to be published.

[10] Khan E, Sandulescu N and Giai NV 2005 Phys. Rev. C 71042801

[11] Grasso M, Khan E, Margueron J and Giai NV 2008 Nucl. Phys. A 8071

[12] Pethick CJ and Ravenhall DG 1995 Annu. Rev. Nucl. Part. Sci. 45429

[13] Barranco F, Broglia RA, Esbensen H and Vigezzi E 1997 Phys. Lett. B390 13

[14] Barranco F, Broglia RA, Esbensen H and Vigezzi E 1998 Phys. Rev. C 581257

[15] Pizzochero PM, Barranco F, Broglia RA and Vigezzi E 2002 Astrophys. J 569381

[16] Sandulescu N, Giai NV and Liotta RJ 2004 Phys. Rev. C 69045802.

[17] Sandulescu N 2004 Phys. Rev. C 70025801

[18] Montani F, May C and Müther H 2004 Phys. Rev. C 69065801

[19] Monrozeau C, Margueron J and Sandulescu N 2007 Phys. Rev. C 75065807

[20] Gandolfi S, Illarionov AY, Fantoni S, Pederiva F and Schmidt KE 2008 Phys. Rev. Lett. 101 132501

[21] Gandolfi S, Illarionov AY, Pederiva F, Schmidt KE and Fantoni S 2009 Phys. Rev. C 80045802

[22] Gezerlis A and Carlson J 2008 Phys. Rev. C 77032801

[23] Schwenk A, Friman B and Brown GE 2003 Nucl. Phys. A 713191

[24] Sedrakian A and Clark JW 2009 in Pairing in fermionic systems, World Scientific, eds. A. Sedrakian, J.W. Clark and M. Alford, p.135

[25] Cao LG, Lombardo U and Schuck P 2006 Phys. Rev C 74064301

[26] Barranco F, Broglia RA, Colò G, Gori G, Vigezzi E and Bortignon PF 2004 Eur. Phys. J. A 21 57.

[27] Pastore A, Barranco F, Broglia RA and Vigezzi E 2008 Phys. Rev. C 78024315

[28] Lesinki T, Duguet T, Bennaceur K and Meyer J 2009 Eur. Phys. J. A 40121

[29] Hebeler K, Duguet T, Lesinski T and Schwenk A 2009 Phys. Rev. C 80044321

[30] Baldo M, Saperstein EE, and Tolokonnikov SV 2007 Eur. Phys. J A 3297

[31] Gori G, Ramponi F, Barranco F, Broglia RA, Co1ò G, Sarchi D and Vigezzi E. 2004 Nucl. Phys. A 731401

[32] Baroni S, Pastore A, Barranco F, Broglia RA and Vigezzi E 2008 arXiv:0805.3962

[33] Magierski P 2004 Int. Jou. Mod. Phys. E 13371

[34] Yakovlev DG and Pethick CJ 2004 Annu. Rev. Astron. and Astrophys. 42169

[35] Shternin PS, Yakovlev DG, Haensel P and Pothekin AY 2007 Mon. Not. R. Astron. Soc. 382 L43

[36] Brown EF and Cumming A, 2009 Astrophys. J. 6981020

[37] Yu Y and Bulgac A 2003 Phys. Rev. Lett. 90161101 
[38] De Blasio FV and Elgaroy Ø 1999 Phys. Rev. Lett. 82815

[39] Elgaroy Ø and De Blasio FV Astron. Astrophys. 2001370939

[40] Avogadro P, Barranco F, Broglia RA and Vigezzi E 2007 Phys. Rev. C 75 012805(R)

[41] Avogadro P, Barranco F, Broglia RA and Vigezzi E 2008 Nucl. Phys. A 811378

[42] Anderson PW and Itoh N 1975 Nature 25625

[43] Lyne AG, Shemar SL and Graham Smith F 2000 Mon. Not. R. Astron. Soc. 315534

[44] Epstein RI and Baym G 1988 Astrophys. J. 328680

[45] Donati P and Pizzochero PM 2004 Nucl. Phys. A 742363

[46] Donati P and Pizzochero PM 2006 Phys. Lett. B 64074

[47] Link B 2009 Phys. Rev. Lett. 102131101 